\title{
Tracking COVID-19 with wastewater
}

\author{
Wastewater testing captures the rise and fall of novel coronavirus cases in a mid-sized metropolitan region.
}

\author{
David A. Larsen and Krista R. Wigginton
}

U nderstanding the full extent of the COVID-19 pandemic is an ongoing challenge for public-health officials. Any epidemiological indicator has biases and limitations. Diagnostic testing capacity may be insufficient; hospitalizations lag infections by weeks and do not report on people with mild or asymptomatic disease. Experience with other viral diseases has shown that monitoring sewage for traces of a pathogen enables effective surveillance of entire communities, providing a sensitive signal of whether the pathogen is present in the population and whether transmission is increasing or declining. Researchers around the world are now pursuing the same approach for COVID-19 with the hope that wastewater data can supplement current measures of its prevalence. The novel coronavirus, SARS-CoV-2, has already been detected in wastewater ${ }^{1,2}$. In this issue, Peccia et al. ${ }^{3}$ demonstrate that concentrations of SARS-CoV-2 RNA in primary sewage sludge tracked COVID-19 cases and hospital admissions during the early weeks of the outbreak in the New Haven, Connecticut, area. Departing from traditional methods of examining wastewater, they report a high-resolution dataset generated from sewage sludge rather than influent and apply statistical analysis to infer the lead time their data may provide over epidemiological indicators. Their results strengthen the evidence that wastewater monitoring could be a powerful tool in tracking the spread of COVID-19.

Wastewater surveillance of COVID19 could have many benefits. It is a cost-effective way to survey transmission dynamics of entire communities. It avoids the biases of other epidemiological indicators ${ }^{4}$. It collects data from people who lack access to healthcare. And if it were successful in revealing infection dynamics earlier than diagnostic testing, it could provide public-health officials with near-real-time information on disease prevalence (Fig. 1).

Wide application of wastewater surveillance began in the 1990s with efforts to eradicate poliovirus ${ }^{5}$. As large-scale vaccination reduced polio transmission, the conventional approach to monitoring polio - tracking cases of acute flaccid paralysis - proved incapable of preventing outbreaks. Because poliovirus infections often present with non-specific symptoms, and acute flaccid paralysis occurs in only one of 200 cases, the virus is able to spread undetected in areas where it was thought to be eliminated. Testing sewage for poliovirus RNA is four to five times more sensitive in detecting outbreaks than monitoring communities for an atypical increase in cases of acute flaccid paralysis and has allowed entire communities to be continuously monitored ${ }^{6}$. When poliovirus was detected in wastewater, mop-up vaccination campaigns prevented new cases of paralysis ${ }^{7}$.

Unlike polio, which spreads mainly by the fecal-oral route, COVID-19 is fueled by respiratory droplets. Yet scientists noted early on that SARS-CoV-2 RNA is frequently detected in patient stool samples. This was not entirely surprising since, during the SARS coronavirus epidemic of 2003, SARS-CoV-1 RNA was found in human feces and in hospital wastewater. The presence of SARS-CoV-2 RNA in feces stems from the ability of the virus to infect ACE2-expressing cells in the small intestine ${ }^{8}$. Despite claims to the contrary ${ }^{9}$, the fecal-oral route is unlikely to be a major factor in the pandemic. Released SARS-CoV-2 viruses are rapidly inactivated in the gastrointestinal-tract fluid and appear to be excreted primarily in a non-infective state. Concentrations of viral RNA in feces vary from patient to patient and over the course of the illness, but the signal can be detected for up to several weeks ${ }^{10}$.

After excretion in feces, the viruses are diluted first in toilet water and then in other municipal wastewater constituents, including graywater (for example, from showers and washing machines) and, in some cases, industrial wastewaters and storm waters. The viruses and their RNA travel through complex sewage systems and can be exposed to different temperatures and chemicals. Viral RNA appears to be stable over the temperatures and time frames involved in travel through the sewage system and settlement in primary wastewater treatment ${ }^{11}$. The signals from the small RNA regions that are targeted in SARS-Cov-2 PCR methods ( $\sim 100$ bases) are likely to long outlast the intact virions and RNA genomes.

It is not currently possible to directly convert concentrations of viral RNA in wastewater to disease prevalence in a community. First, the biological variability in viral RNA excretion over time and between individuals creates problems in this estimate. This variability is then compounded by variability in the sewer systems across communities, particularly their size, configuration, and whether they include stormwater and industrial waste. However, longitudinal trends of SARS-CoV-2 RNA levels in wastewater can still be helpful in complementing traditional surveillance methods to understand trends in community transmission.

Most early studies of SARS-CoV-2 RNA in wastewater have focused on wastewater influent - water that enters the treatment plant. In contrast, Peccia et al. collected daily samples from sludge, the solids that settle during the first steps of municipal wastewater treatment. After extracting nucleic acids directly from small volumes of mixed sludge samples, the authors used reverse transcription quantitative $\mathrm{PCR}$ (RT-qPCR) to quantify both the N1 and N2 gene targets of SARs-CoV-2. They detected SARS-CoV-2 RNA in all collected samples, with concentrations ranging from $1.7 \times 10^{3}$ to $4.6 \times 10^{5}$ virus RNA copies per milliliter of primary sludge. Compared to non-enveloped viruses, coronaviruses have an affinity for wastewater solids ${ }^{12}$; therefore, sludge monitoring at the community scale may offer greater sensitivity and less sample variance compared with wastewater influent monitoring.

Peccia et al. studied the New Haven metropolitan area (population 200,000) over an initial wave of COVID-19 cases, from 19 March to 1 June 2020, when reported daily new (non-averaged) cases rose from near 0 to as high as $\sim 150$ and then declined to under 25. Comparing their sludge results with publicly available 


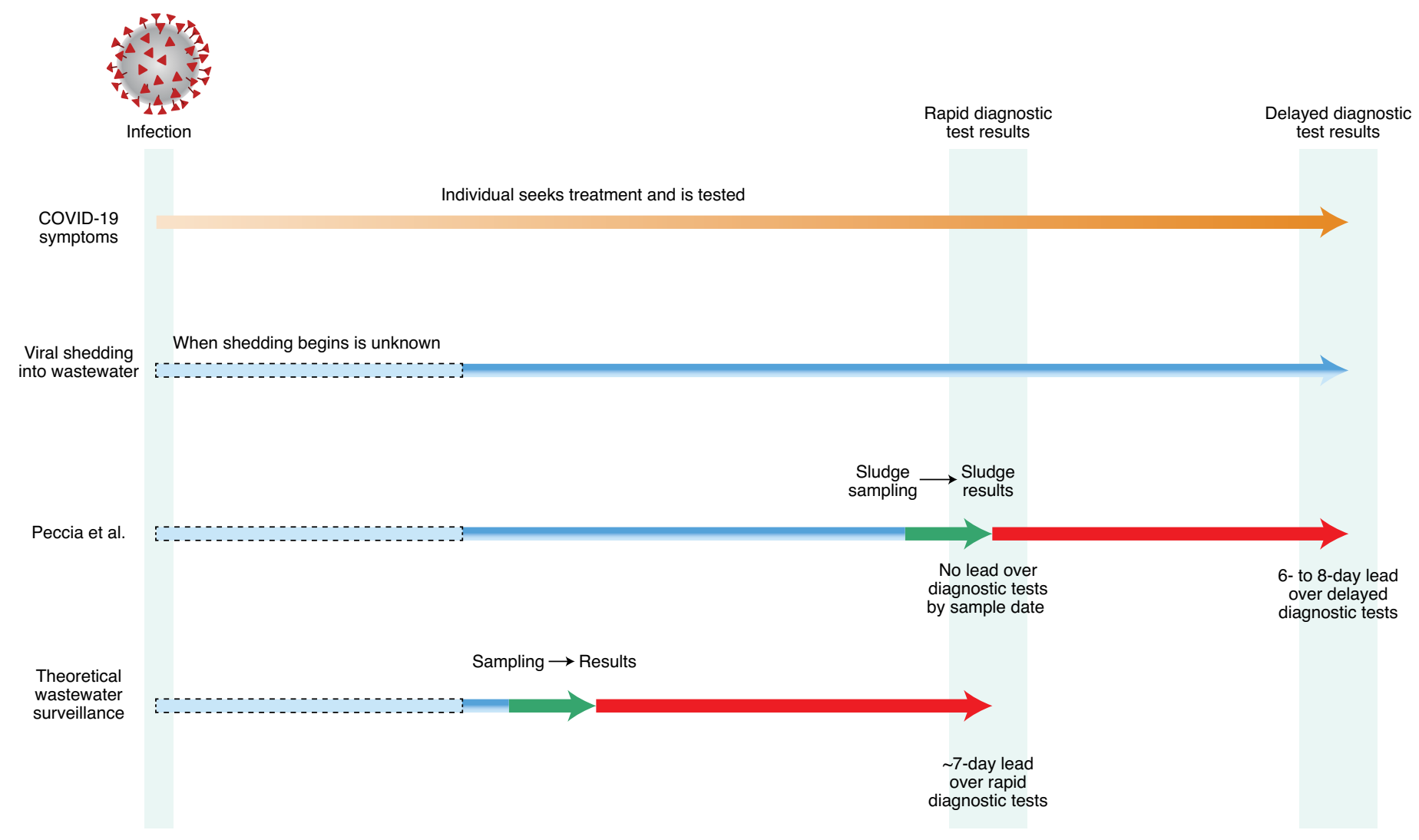

Fig: 1 | Observed and theoretical time lags between infection and detection of increasing SARS-CoV-2 transmission in wastewater and the health system.

data, they found that the trends in sludge SARS-CoV-2 RNA concentrations matched the trends of new COVID-19 cases and hospitalizations. Despite the relatively large variance (noise) in the sludge data, the high-resolution daily samples allowed the authors to establish a trend line, which correlated with reported cases. Weekly or even semiweekly sludge samples may not have been as informative.

An intriguing finding of this study is that sludge RNA gave early warning of epidemiological trends only when delays in diagnostic test processing were taken into account (Fig. 1). Theoretically, we expect wastewater surveillance to lead diagnostic tests by perhaps a week given what we know about viral dynamics in individuals and fecal shedding. Shedding may occur soon after infection, whereas an infected person identified through the health system must develop symptoms and seek treatment before receiving a diagnosis. The finding of Peccia et al. that sludge RNA lacks predictive power compared with diagnostic tests (excluding delays in test processing) raises questions about the early-warning theory. However, epidemiological data in the early days of the pandemic were fraught with limitations, including limits on testing capacity and changes in testing guidance over time. Before we abandon the prospect of wastewater as an early-warning system, further research with more robust epidemiological data is needed to better assess its potential. Moreover, in communities where testing delays persist, the early-warning value of wastewater surveillance is already clear.

Another benefit of wastewater surveillance is that it lacks the biases of the traditional indicators used to understand where disease transmission is occurring, increasing, or decreasing. In the early days of the pandemic, a key indicator was the cumulative number of diagnosed cases. Later, more attention was given to hospitalizations, deaths and, most recently, rates of test positivity and serologic data. These indicators, while useful, have biases ${ }^{4}$. For example, the number of cases depends on access to diagnostics, which has been limited during the pandemic, and the threat of isolation and quarantine can dissuade people from getting tested. Hospitalizations and deaths lag transmission by weeks. Like total cases, rates of test positivity depend on testing regimens, protocols and availability.

These biases are absent in wastewater surveillance. Daily sampling of SARS-CoV-2
RNA in wastewater would provide information similar to that from daily random testing of hundreds of individuals in a community, but it is more cost-effective and less invasive. Wastewater data could also be used to check the reliability of epidemiological trends calculated from diagnosed cases.

Much more research is needed to understand how SARS-Cov-2 detected in wastewater corresponds to COVID-19 transmission, and especially whether it can act as an early-warning system. This research should consider such variables as diverse transmission dynamics, diverse geographic areas and different solids collection processes at wastewater treatment facilities. It will also be important to compare influent and sludge from the same wastewater treatment plant to determine which approach is more sensitive to low case numbers in a population.

Sludge sampling is relatively straightforward to implement in wastewater treatment plants, where large composites of sludge are collected. However, obtaining sludge samples is more complicated upstream of wastewater treatment plants or in local settings, such as prisons, residences, dormitories and nursing homes. It could not 
be performed easily throughout a sewershed to disaggregate city-wide trends.

The next steps in refining wastewater monitoring tools include operationalizing the technology into a useful surveillance network. Many groups are already working on this, including New York State's SARS2-EWSP, Utah State's SARS-CoV-2 Sewage Monitoring (https:// deq.utah.gov/water-quality/sars-cov2-sewage-monitoring) and Tempe, Arizona's COVID-19 Wastewater Results (https:// covid19.tempe.gov). Peccia et al. collected frozen daily sludge samples once per week, and their conclusions assume that wastewater solids are analyzed and reported on the day they were collected. Any delay in sludge sample processing would erode the potential lead time of this approach over traditional epidemiological indicators.
Unlike with polio, public-health officials cannot yet respond to an increase in cases with a vaccine. But when vaccines become available, wastewater surveillance could speed their deployment to areas where upticks are detected. Even now, it could facilitate social distancing interventions before community transmission reaches exponential growth and could help governments mitigate the socioeconomic consequences of extended lockdowns.

David A. Larsen $1{ }^{1,3 凶}$ and
Krista R. Wigginton $2,3 \bowtie$
${ }^{1}$ Department of Public Health, Syracuse University,
Syracuse, NY, USA. ${ }^{2}$ Department of Civil and
Environmental Engineering, University of Michigan,
Ann Arbor, MI, USA. ${ }^{3}$ These authors contributed
equally: David A. Larsen, Krista Wigginton.
$\bigotimes_{\text {e-mail: dalarsen@syr.edu; kwigg@umich.edu }}$

David A. Larsen (D) 1,3凶 and

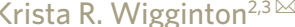

Syracuse, NY, USA. ${ }^{2}$ Department of Civil and

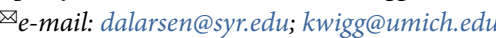

Published online: 21 September 2020

https://doi.org/10.1038/s41587-020-0690-1

References

1. Medema, G., Heijnen, L., Elsinga, G., Italiaander, R. \& Brouwer, A. Environ. Sci. Technol. Lett. 7, 511-516 (2020).

2. Randazzo, W. et al. Water Res. 181, 115942 (2020).

3. Peccia, J. et al. Nat. Biotechnol. https://doi.org/10.1038/s41587020-0684-z (2020).

4. Rowe, A. K. et al. Malar. J. 8, 209 (2009).

5. Asghar, H. et al. J. Infect. Dis. 210, S294-S303 (2014).

6. Kroiss, S. J. et al. PLoS ONE 13, e0208336 (2018).

7. Brouwer, A. F. et al. Proc. Natl Acad. Sci. USA 115, E10625E10633 (2018).

8. Zang, R. et al. Sci. Immunol. 5, eabc3582 (2020).

9. Bogler, A. et al. Nat. Sustain. https://doi.org/10.1038/s41893-02000605-2 (2020).

10. Wölfel, R. et al. Nature 581, 465-469 (2020)

11. Ahmed, W. et al. Environ. Res. https://doi.org/10.1016/j. envres.2020.110092 (2020).

12. Ye, Y., Ellenberg, R. M., Graham, K. E. \& Wigginton, K. R. Environ. Sci. Technol. 50, 5077-5085 (2016).

Competing interests

The authors declare no competing interests.

\section{PODCAST}

\section{First Rounders: Noubar Afeyan}

Noubar Afeyan is the founder and CEO of Flagship Pioneering. His conversation with Nature Biotechnology covers his family fleeing the Lebanese Civil War when he was a boy, how a chance encounter at a scientific meeting opened his mind to entrepreneurism, and why immigrants (and entrepreneurs) benefit from having a "paranoid optimist" mindset.

https://play.acast.com/s/naturebiotechnologypodcast/noubarafeyan

Published online: 5 October 2020

https://doi.org/10.1038/s41587-020-0683-0 